\title{
Precise Measurement of Vertebral Bone Density Using Computed Tomography Without the Use of an External Reference Phantom
}

\author{
Scott D. Boden, David J. Goodenough, Charles D. Stockham, Eugene Jacobs, \\ Thomas Dina, and Robert M. Allman
}

Bone density measurement by quantitative computed tomography (OCT) commonly uses an external reference phantom to decrease scan-to-scan and scanner-to-scanner variability. However, the peripheral location of these phantoms and other phantom variables is also responsible for a measurable degradation in accuracy and precision. Due to non-uniform artifacts such as beam hardening, scatter, and volume averaging, the ideal reference phantom should be as close to the target tissue as possible. This investigation developed and tested a computer program that uses paraspinal muscle and fat tissue as internal reference standards in an effort to eliminate the need for an external phantom. Because of their proximity, these internal reference tissues can be assumed to reflect more accurately the local changes in the $x$-ray spectra and scatter distribution at the target tissue. A user interactive computerized histogram plotting technique enabled the derivation of reproducible CT numbers for muscle, fat, and trabecular bone. Preliminary results indicate that the use of internal reference tissues with the histogram technique may improve reproducibility of scan-toscan measurements as well as inter-scanner precision. Reproducibility studies on 165 images with intentional region-of-interest (ROI) mispositioning of $1.5,2.5$, or $3.5 \mathrm{~mm}$ yielded a precision of better than $1 \%$ for normals and $1 \%$ to $2 \%$ for osteoporotic patients-a twofold improvement over the precision from similar tests using the standard technique with an external reference phantom. Such improvements in precision are essential for QCT to be clinically useful as a noninvasive modality for measurement of the very small annual changes in bone mineral density.

1989 by W.B. Saunders Company

KEY WORDS: QCT, bone density, reference phantom, artifacts, precision, accuracy, osteoporosis.

Q UANTITATIVE computed tomography (QCT) is a clinically established and widely applied technique for noninvasive bone mineral density (BMD) assessment. ${ }^{1,2}$ Recently, the clinical utility of data generated using this method has been questioned. ${ }^{3,4}$ Considering the physiologic rate of trabecular bone loss in women is approximately $1.2 \% / \mathrm{yr}$, the current reported precision for QCT measurements (3\% to 5\%) does not permit reliable measurement of such small changes at annual intervals. ${ }^{5}$ For example, a method with $2 \%$ reproducibility must demonstrate a change of $5.7 \%$ between measurements to maintain a $95 \%$ confidence level. ${ }^{2}$ Although absolute accuracy is also important, it does not significantly limit the clinical utility of QCT as long as bone density measurements for a patient are compared with previous measurements or control patients measured using the same technique. Actually, it is precision (reproducibility) that represents the limiting factor in the clinical utility of bone density measurements.

In an effort to improve accuracy and reproducibility, a number of phantoms have been developed to offer reference standards and/or methodologies for QCT studies. ${ }^{1,6}$ The early thrust of research in phantom development was directed at correction for CT instability or scanto-scan variations. Although early third generation scanners may have shown considerable scanto-scan variation, it is no longer the case that functional CT scanners show such variations. Over the last decade, one of the authors (DJG, unpublished data) has measured hundreds of CT scanners in the public and private sectors; the variation of intra-scanner $\mathrm{CT}$ numbers is within 1 to 2 Hounsfield units (HU) and is systematic and reproducible. Ironically, the misconception that QCT suffers from considerable scanner drift or variability is partially propagated by examination of external phantoms. When a QCT phantom is located externally to patients, it will necessarily be subject to attenuation from different patient-moderated spectra that are depen-

From the Departments of Radiology and Orthopaedics, The George Washington University Medical Center, Washington, DC and Columbia Scientific, Inc., Columbia, MD.

Presented in part at the Ninth Conference on Computer Applications in Radiology, Hilton Head, SC, June 2, 1988.

Address reprint requests to Scott D. Boden, MD, Department of Orthopaedic Surgery, George Washington University Medical Center, 2150 Pennsylvania Ave, NW, Washington, $D C, 20037$.

(C) 1989 by W.B. Saunders Company.

0897-1889/89/0201-0003\$03.00/0 
dent upon patient composition, size, and geometric position. Accordingly, much of the variation in the CT numbers of the phantom is not due to scanner variability, but to variation in the patient's overlying the phantom. Instability in phantom concentrations and air bubbles in the liquid phantoms have also contributed to this problem.?

There is evidence that use of externally located calibration phantoms results in a measurable degradation in precision due to patient-moderated artifacts (beam hardening and scatter), partial volume averaging, and repositioning errors. ${ }^{8}$ The purpose of this investigation was to study the magnitude of some of these errors and to develop a precise computerized method of BMD measurement without the error introduced by an external calibration phantom.

\section{MATERIALS AND METHODS}

The development and analysis of techniques for vertebral BMD determination used in this study was accomplished by studying 48 healthy volunteers. Patients were scanned on a GE CT/T 8800 or 9800 scanner (General Electric, Milwaukee, WI). Scan parameters for the GE 8800 were $120 \mathrm{kVp}$ tube potential with $5-\mathrm{mm}$ thick slices, and $140 \mathrm{kVp}$ with 5-mm slices for the GE 9800. Patients were scanned while lying on top of a commercially available external calibration phantom containing tubes of water, ethanol, and several concentrations of $\mathrm{K}_{2} \mathrm{HPO}_{4}$ (Image Analysis, Irvine, CA). Scan slices were obtained through the middle portion of the $\mathrm{L} 1$ to $\mathrm{L} 4$ vertebral bodies parallel to the endplates as described by Cann et al. ${ }^{5}$ BMD was calculated for all subjects at each lumbar level using two methods: (1) BMD was calculated with the external calibration phantom using the commercially available software (Image Analysis). The CT numbers of the three tubes of $\mathrm{K}_{2} \mathrm{HPO}_{4}$ present on each cut were plotted against their known concentrations to construct a standard curve. The vertebral BMD was then extrapolated from this curve by measuring the mean CT number of a standardized elliptical region of interest (ROI) manually positioned in the trabecular portion of the vertebral body (Fig 1). A composite bone density was calculated from the average density of each vertebra scanned; and (2) BMD was calculated without using the external calibration phantom. A computer program developed for this investigation used a histogram technique for identifying and measuring the CT number of a tissue of interest. A large ROI was placed posterior to the lumbar vertebrae and its histogram was calculated (Fig 2). The histogram measured the frequency of occurrence of each CT number within the ROI Paraspinal muscle and surrounding fat tissues produced two isolated peaks within the histogram distribution that were identified using a computer generated gradient search and least squares fitting algorithm. The CT number of the two tissues of interest was defined as the central value of the best fit to the most probable normal Gaussian distribution of their peaks

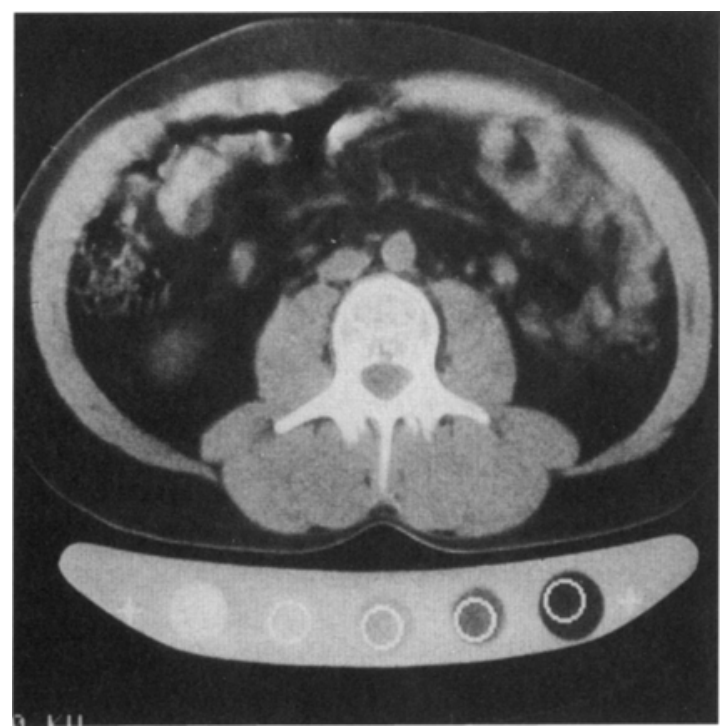

Fig 1. Standard method for QCT bone density measurement using an external reference phantom beneath the patient for calibration of CT numbers with bone density in $\mathrm{mg} / \mathrm{cc} \mathrm{K}_{2} \mathrm{HPO}_{4}$ equivalents.

within the histogram plot. This definition differs from the conventionally used mean CT number. This is the mode (most frequently occurring value) of the $\mathrm{CT}$-number distribution of the muscle and fat tissue within the ROI.

A second, smaller ROI was positioned to circumscribe the

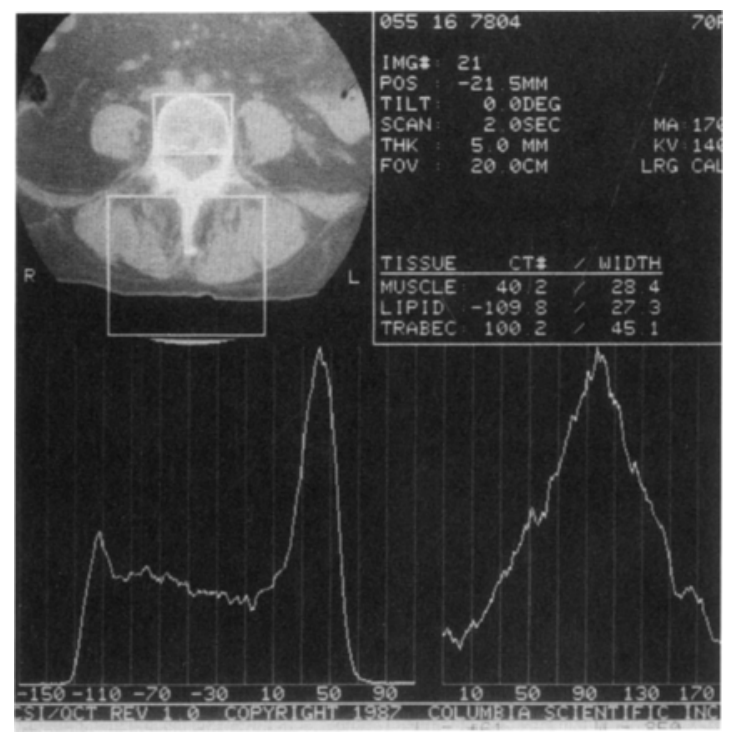

Fig 2. Histogram technique for QCT bone density measurement without an external calibration phantom. The histogram plot indicates the mode of the CT number distribution for specific tissues. Paraspinal muscle and fat from the larger ROI produce the two histogram peaks (left) and are used as an internal reference standard to translate the CT number of trabecular bone (single peak, right) to $\mathrm{mg} / \mathrm{cc}$ bone density equivalents. 
vertebral trabecular bone to be measured. This region is dominated by trabecular bone and marrow tissue producing an isolated, but broader peak in the histogram distribution. The ROI need not exclude cortical bone; this bone peak would be at the high end of the histogram and would be clearly distinct from the trabecular bone or reference tissue peaks. The histogram peak for trabecular bone was identified using similar computer algorithms that were used for the reference tissue peaks. By using the muscle and fat CT numbers as internal reference standards to generate a standard curve, the CT number for trabecular bone was then used to extrapolate an equivalent bone density $(\mathrm{mg} / \mathrm{cc})$. The densities from multiple lumbar vertebrae in each patient were averaged to yield a composite BMD.

A series of experiments were performed to study the effects of scanner variation, ROI positioning, air gap artifact, and beam hardening on vertebral BMD measurements.

(1) The effect of variable air gap between the patient and the external calibration phantom was studied. In 20 patients, the CT number of the water tube in the external reference phantom was compared with the width of the air gap.

(2) The effect of beam hardening due to variation in patient size and composition was studied. In 20 patients the $\mathrm{CT}$ number of the $200 \mathrm{mg} / \mathrm{cc} \mathrm{K}_{2} \mathrm{HPO}_{4}$ tube in the external reference phantom was compared with the patient thickness.

(3) The effect of scanner calibration file size on BMD was studied. Data averaged from 28 slices of 11 patients (medium size calibration file) were compared with 21 slices of eight patients (larger calibration file). All patients were scanned with water bags filling the gap between the patient and the external reference phantom. The equivalent bone density of the water in the spacer bags was calculated using both the standard and the histogram techniques. Comparison was made between the CT numbers on scans done with the large calibration file and scans performed with the medium file.

(4) Scanner-to-scanner variability was studied. Data from 28 slices of 11 patients scanned on a GE 8800 were compared with 41 slices of 18 patients studied on a different scanner of the same model. The same external calibration phantom was transported between locations so that all scans used the same identical phantom. The predicted equivalent bone density of the water tube in the external phantom was calculated using the external reference standard and again using the internal tissue reference standards.

(5) The effect of ROI positioning was studied for the histogram technique. A set of lumbar spine scans from two scanners (seven images-GE 9800, 26 images-GE 8800) were analyzed retrospectively. The ROI was selected, then moved off center by varying amounts $(1.56,2.56$, or $3.12 \mathrm{~mm})$ to the left, right, up, and down. CT numbers for muscle, fat, and trabecular bone were calculated in each position using the histogram technique. The error in precision of the calculated CT number was the sample standard deviation of the five measurements made on each image.

(6) The correlation of bone density measured by the histogram technique to the method of Cann and Genant was studied. For each image in all patients scanned, the BMD in $\mathrm{mg} / \mathrm{cc} \mathrm{K}_{2} \mathrm{HPO}_{4}$ from the standard technique (using the external reference phantom) was compared with $\mathrm{mg} / \mathrm{cc}$ of equivalent bone density that was calculated using the histogram technique without the use of the external reference phantom.

\section{RESULTS}

The effect of a variable air gap between the patient and the external reference phantom was examined by comparing the width of the air gap with the CT number for the water component of the phantom. Increased air gap correlated strongly with a decreased CT number for the water section of the phantom (slope $=-0.67$ $\mathrm{HU} / \mathrm{mm}$ air gap, $r=0.999)$. A variable air gap of only 2 to $3 \mathrm{~mm}$ in repeat studies would produce a 1 to $2 \mathrm{CT}$ number error in the water reference standard (Fig 3).

A moderate correlation was found between patient thickness and the CT number of the 200 $\mathrm{mg} / \mathrm{mL} \mathrm{K}_{2} \mathrm{HPO}_{4}$ component of the external reference phantom $(r=0.905)$. A variation in patient thickness of $5 \mathrm{~cm}$ could result in a 10 to 15 HU shift in the reference phantom CT number (Fig 4).

The GE 8800 and 9800 CT scanners provide a choice of two body calibration files (medium and large) to correct for patient sizes. There was a substantial inter-patient deviation in CT numbers between the large and medium calibration file groups (Table 1). CT numbers of various reference tissues were shifted by an average of

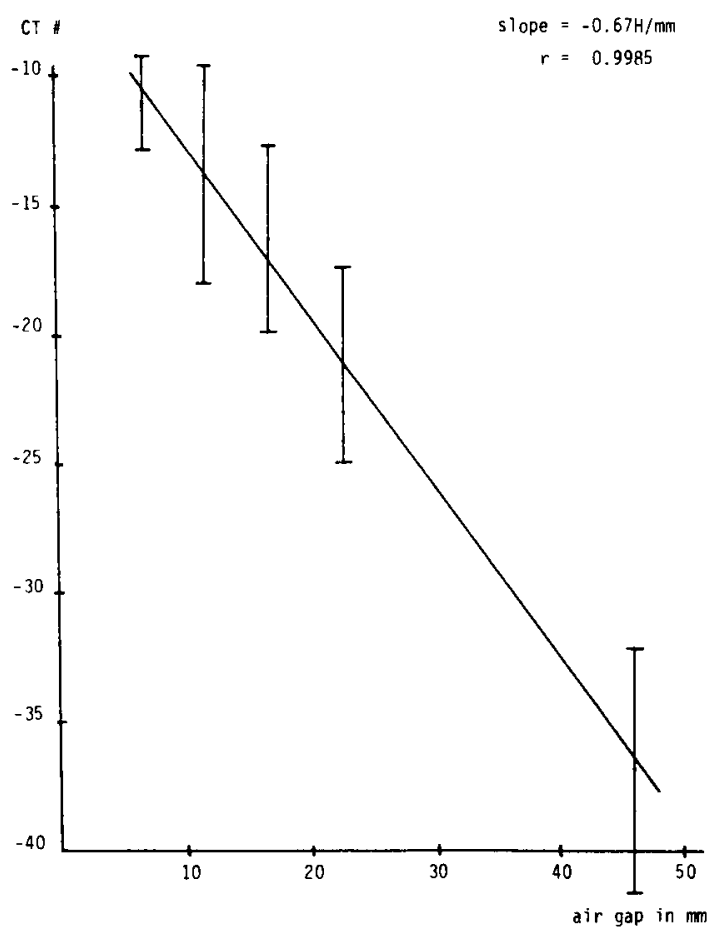

Fig 3. Relationship of patient to phantom air gap and the CT number of the water component of the external reference phantom. A linear relationship is demonstrated. 


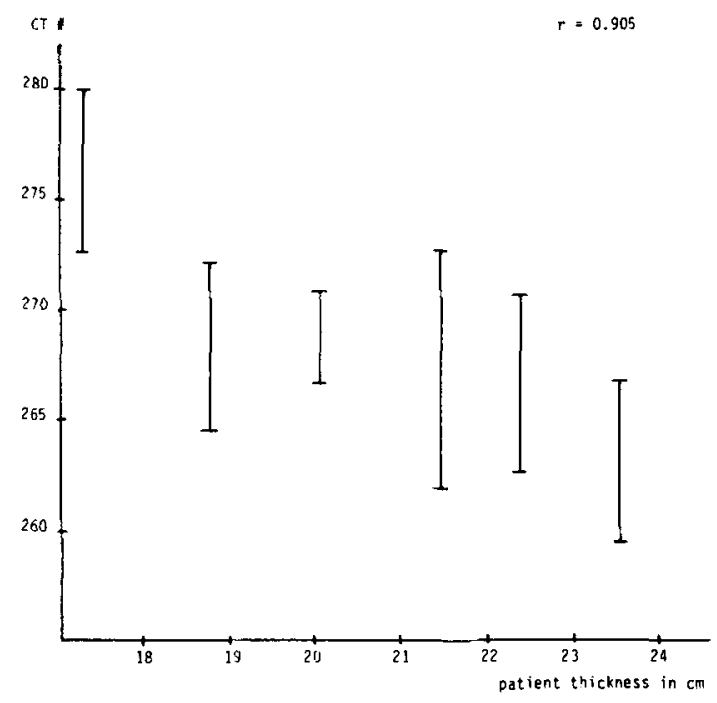

Fig 4. Relationship of patient thickness and the CT number of the $200 \mathrm{mg} / \mathrm{mL} \mathrm{K}_{2} \mathrm{HPO}$, component of the external reference phantom. A significant downward shift in CT number is seen with larger patients due to nonuniform beam hardening and scatter artifacts.

12.2 HU when changing from a medium to a large calibration file. There was no difference in the equivalent bone density of the water spacer predicted by the histogram technique. There was a slightly larger disparity in the predicted bone density using the conventional technique between the medium and large calibration file groups. More importantly, the sample standard deviations for the histogram technique were smaller than the standard technique.

Comparison of the absolute CT numbers for various reference tissues on two different GE 8800 scanners showed a significant variation. The CT numbers were shifted an average of 15.0 HU from one scanner to the other (Table 2). The scanner-to-scanner difference in equivalent bone density for the water component of the external phantom was minimal as predicted by both of the QCT methods studied. However, the sample standard deviations were smaller than the con- ventional technique predictions that used the external reference phantom.

The effect of ROI repositioning on bone density determined by the histogram technique was relatively small (Table 3 ). The average error in precision was $<1 \mathrm{HU}$ for muscle and fat reference tissues. The error in reproducibility of the trabecular bone CT number varied from 1 to 2 HU for ROI shifts of 1.5 to $2.5 \mathrm{~mm}$.

As an estimate of accuracy, the BMD of all patients, which was calculated using the external reference phantom, was compared with the equivalent bone density using the histogram technique (Fig 5). Although the absolute bone density was slightly higher when calculated by the external phantom method, the correlation of the two techniques was strong $(r=0.96)$.

\section{DISCUSSION}

Early in the evolution of computed tomography, there was considerable debate concerning the quantitative capability of $\mathrm{CT}^{9,10}$ Continued interest in evaluation of osteoporosis and other metabolic diseases has generated a great deal of research in ways to improve the accuracy and precision of CT. ${ }^{11}$ Reference phantoms have been used in an attempt to correct in vivo problems that lead to CT number variability of a given tissue type when situated in a heterogeneous surround of other tissues and attenuating media (including bone, muscle, and fat). The effects of these other tissues contribute to the well-known errors of beam hardening, scatter, and partial volume averaging. ${ }^{12}$

Due to differences in geometry and transmission path lengths, externally located phantoms are subject to considerably different spectra and scatter distribution than the centrally located vertebral bodies that are being measured. Beam hardening affects the accuracy and precision of bone density measurements because the energy

Table 1. Effect of Calibration File Size on Mean CT Number of Various Tissues

\begin{tabular}{lcr}
\hline \multicolumn{1}{c}{ Tissue } & Large File $(\mathrm{n}=21)$ & Medium File $(\mathrm{n}-28)$ \\
\hline Retrospinal fat & $-93.4 \pm 12.9$ & $-107.7 \pm 19.4$ \\
Paraspinal muscle & $66.9 \pm 10.4$ & $54.8 \pm 11.7$ \\
Water tube of phantom & $10.9 \pm 10.1$ & $0.9 \pm 12.0$ \\
Water spacer (ERP)* & $5.5 \pm 3.1 \mathrm{mg} / \mathrm{cc}$ & $4.6 \pm 5.2 \mathrm{mg} / \mathrm{cc}$ \\
Water spacer (Histo)* & $-3.6 \pm 2.7 \mathrm{mg} / \mathrm{cc}$ & $-3.4 \pm 4.3 \mathrm{mg} / \mathrm{cc}$ \\
\hline
\end{tabular}

-Equivalent bone density of the water spacer bags calculated by use of the external reference phantom (ERP) calibration data or by the histogram/internal reference tissue method (histo). 
Table 2. Effect of Intra-Scanner Variability on Mean CT Number of Various Tissues

\begin{tabular}{lcc}
\hline \multicolumn{1}{c}{ Tissue } & GE $8800 \# 1$ (n - 28) & GE $8800 \# 2$ (n - 41) \\
\hline Retrospinal fat & $-107.7 \pm 19.4$ & $-122.2 \pm 8.0$ \\
Paraspinal muscle & $54.8 \pm 11.7$ & $38.4 \pm 6.1$ \\
Water tube of phantom & $0.9 \pm 12.0$ & $-13.3 \pm 3.8$ \\
Water tube of phantom (ERP)* & $-6.5 \pm 2.3 \mathrm{mg} / \mathrm{cc}$ & $-6.0 \pm 2.5 \mathrm{mg} / \mathrm{cc}$ \\
Water tube of phantom (Histo) * & $-9.0 \pm 5.8 \mathrm{mg} / \mathrm{cc}$ & $-9.2 \pm 4.7 \mathrm{mg} / \mathrm{cc}$ \\
\hline
\end{tabular}

"Equivalent bone density of the water tube of the external phantom as calculated using the external reference phantom (ERP) calibration data or by the histogram/internal reference tissue method (Histo).

spectrum of the $\mathrm{x}$-rays passing through the vertebral trabecular bone is shifted toward higher energies as compared with the energy spectrum of the beam passing through either the soft tissues or the external reference phantom. ${ }^{13-15}$ Scattered radiation introduces non-uniform CT number shifts over the scan field with magnitude dependent on patient size, shape, and position in the scan field. ${ }^{8}$ Use of an external reference phantom heightens these problems because of its peripheral location and its position relative to the patient is not fixed. Some of the disadvantages of an external calibration phantom were demonstrated in this investigation by the CT number shifts caused by the air gap artifact and variable patient size, confirming similar reports in the literature. ${ }^{16,17}$ To best correct these and other related effects, the ideal reference phantom would be placed at the site of the tissue being measured.

Clearly, a reference phantom cannot be inserted into the patient. However, this investigation has shown that certain intrinsic tissue samples, namely fat and muscle, offer reliable internal reference standards. These tissues can generally be found close enough to the target tissue to minimize the effects of physical factors such as beam hardening, scatter, and volume averaging that are exacerbated by the peripheral location of external reference phantoms. In addition, reference tissues that are "linked" to the patient and maintain their relative position with the vertebral trabecular bone will diminish the reproducibility errors associated with "nonlinked" external reference phantoms.

There are many theoretic advantages for using internal tissues as references for correcting scanto-scan and scanner-to-scanner variations as well as in vivo artifacts accentuated by the external calibration phantoms. Until now, there has not been a method for accurate and reproducible measurement of soft tissue CT numbers. Soft tissues are distributed throughout a CT slice in an unpredictable and non-uniform fashion, typically with varying degrees of intermixing so that it is impossible to reliably locate a conventional $\mathrm{ROI}$ for calculating a mean $\mathrm{CT}$ number.

The user interactive computer program developed for this investigation uses a histogram plotting technique for identifying and measuring the CT number of a specific tissue of interest. Determination of mode 1 of the CT number distribution minimizes the problems with tissue intermixing. The histogram peaks calculated by the software represent a measurement close to the CT numbers of pure muscle and fat. Image pixels with various mixtures of these and other soft tissues below the resolution of the scanner will fall between the two peaks. The sharpness of the peak and its effective resolution from the background distribution is determined by the relative concentration of the tissue and the physical factors mentioned earlier. Fat and skeletal muscle have fixed chemical compositions and known linear attenuation coefficients as a function of $\mathrm{x}$-ray energy that allow them to be used as reference tissues to plot a standard calibration curve. ${ }^{18}$ The axial orientation of the paraspinal

Table 3. Effect of Intentional ROI Repositioning on the CT Number of Various Tissues Using the Histogram Technique

\begin{tabular}{lcccc}
\hline & \multicolumn{2}{c}{ GE $9800(n-35)$} & & GE $8800(\mathrm{n}-130)$ \\
\cline { 2 - 3 } ROI shift & $1.56 \mathrm{~mm}$ & $3.12 \mathrm{~mm}$ & & $2.56 \mathrm{~mm}$ \\
\hline Muscle & $P=0.1 \mathrm{HU}$ & $P=0.2 \mathrm{HU}$ & & $P=0.3 \mathrm{HU}$ \\
Fat & $P=0.1 \mathrm{HU}$ & $P=0.2 \mathrm{HU}$ & & $P=0.7 \mathrm{HU}$ \\
Trabecular & $P=1.2 \mathrm{HU}$ & $P=2.5 \mathrm{HU}$ & & $P=2.2 \mathrm{HU}$ \\
\hline
\end{tabular}

$P$, the error in precision of the calculated CT number defined as the standard deviation of five measurements made with the ROI retrospectively shifted the specified magnitude in four different directions on each image.

HU, Hounsfield units. 


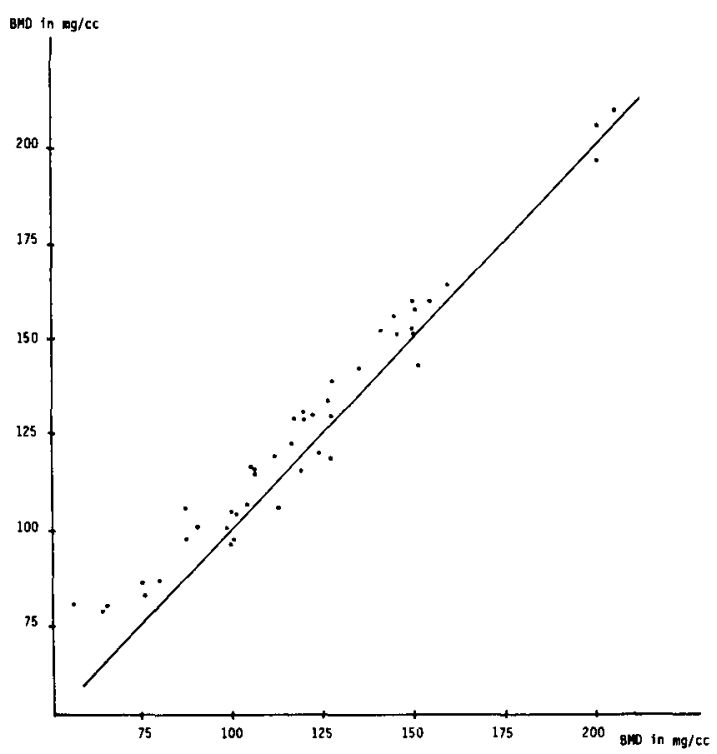

Fig 5. Correlation of bone density calculated on the same patients by the histogram technique using internal reference standards ( $x$-axis) and by the external reference phantom technique (y-axis). A strong correlation is seen $(r=0.96)$.

muscles and surrounding fat that travel longitudinally for the length of the spine virtually eliminates the effects of axial plane partial volume averaging. ${ }^{19}$

The concept of increased artifact and decreased precision with reference standards located further away from the spine was illustrated by the investigation of the ability of two QCT methods to correct for calibration file changes and scanner differences. The effects of changing the GE scanner calibration file appeared to decrease the precision with the histogram technique less than with the external phantom method. Analysis of scanner-to-scanner variability showed both techniques corrected well for scanner differences, but in the calibration file experiment, the histogram technique demonstrated slightly poorer precision. To understand this phenomenon, one must recall that the calibration file experiment compared measurements of the water spacers (relatively close to the spine), while the intra-scanner comparison studied measurements of the water tube in the external phantom (far from the spine). The use of internal reference tissues corrected for these variables at least as well as the external phantom method. However, while the external reference phantom showed greater precision for predicting the density of a tissue nearby (ie, the water tube of the phantom), the internal reference tissues demonstrated better precision in measuring tissues closer to them (ie, the water spacers). These results support the concept that the ideal reference standard should be as close as possible to the tissue of interest being measured.

This investigation has suggested that the use of internal reference tissues close to the vertebral bone being measured will decrease QCT reproducibility errors caused by beam hardening and scatter. However, scan-to-scan reproducibility is also dependent on patient relocalization precision within the scanner and the error in relocation of the ROI used to measure the CT number of the reference and trabecular bone. ${ }^{20}$ In fact, it has been estimated that repositioning the ROI around the same volume of trabecular bone may be responsible for as much as $50 \%$ of the overall reproducibility error. ${ }^{6}$

CT slice positioning is usually performed using a computerized radiographic localization system. Slice localization (and patient repositioning) errors along the longitudinal axis have been measured for the GE 9800 and 8800 scanners as 0.5 to $1.0 \mathrm{~mm} .^{16,21}$ With this measured reproducibility, $90 \%$ or more of a given $10-\mathrm{mm}$ thick section will be scanned in serial studies. ${ }^{21}$ The tissue density of the lumbar vertebral body is fairly uniform (1 to $2 \mathrm{HU} / \mathrm{mm}$ shift) in the central 13 to $15 \mathrm{~mm}$ between the endplates, so that even a $2-\mathrm{mm}$ error in serial studies contributes $<2 \%$ error to reproducibility. ${ }^{21}$

The remainder of scan-to-scan reproducibility is largely dependent on reselection of the reference tissue and target tissue ROIs. The standard method of ROI selection is to inscribe the ROI within the target tissue and determine the mean CT number. ROI positioning errors are magnified if the target tissue is not homogeneous. The CT number distribution within vertebral trabecular bone is highly non-uniform due to beam hardening and partial volume effects near the cortical shell and the encroachment of basivertebral veins. Using this standard technique, errors of 3 to $4 \mathrm{CT}$ numbers have been reported for ROI shifts as small as $1.5 \mathrm{~mm} .^{12}$ This translates to a reproducibility error of slightly less than $2 \%$ for normals and as high as $3 \%$ to $4 \%$ for osteoporotic patients.

The computer program developed for this 
investigation uses a different technique whereby the ROI circumscribes the target tissue. Histogram analysis determines the mode or most frequently occurring CT number as opposed to the mean CT number. Accordingly, exact placement and size of the ROI are less important with this technique; the larger the ROI, the greater the reproducibility. Reproducibility studies on 165 images with intentional ROI mispositioning of 1.5 to $2.5 \mathrm{~mm}$ yielded a sample standard deviation of 1 to 2 CT numbers for trabecular bone. This translates into a precision of better than $1 \%$ for normals and $1 \%$ to $2 \%$ for osteoporotic patients, a twofold improvement over the precision reported from a similar experiment using the standard QCT technique with an external phantom. ${ }^{12}$

One other factor contributing to error in QCT measurements deserves mention. The variability of trabecular marrow fat content has been implicated as a source of accuracy errors in singleenergy QCT bone density measurements. ${ }^{22,23}$ This inaccuracy has been measured to be as much as $13 \mathrm{mg} / \mathrm{mL} \mathrm{K} \mathrm{K}_{2} \mathrm{HPO}_{4} / 10 \%$ increase in marrow fat content at $130 \mathrm{kVp} .^{22}$ Use of dualenergy QCT techniques can minimize this error, but it is often accomplished at the expense of precision. ${ }^{23}$ Since the changes in marrow fat content appear to be systematic and age-related, some investigators believe that dual-energy corrections are unnecessary if single-energy QCT patients are compared with age-matched controls that are also scanned by single-energy QCT ${ }^{24}$ Regardless, this effect is independent of whether external reference phantoms or internal reference tissues are used for calibration.

The authors emphasize that this investigation has focused on studying the improvement in precision with the new histogram technique. A direct measure of the accuracy of this method of QCT bone densitometry without an external calibration phantom has not yet been undertaken. However, in this investigation a direct comparison was made to an accepted method that has been proven to be accurate ( $5 \%$ to $10 \%$ ) by cadaver experiments verified by ash weights. ${ }^{2}$ This investigation showed a strong correlation between the two methods, and the accuracy of the new techniuqe is expected to be of the same order.

This investigation has provided data relevant to several artifacts and errors that may effect precision of QCT bone density measurements due to the use of an external calibration phantom. Since bone density measurements for a patient are usually compared with previous measurements or to controls measured with the same technique, it is precision (reproducibility), rather than absolute accuracy that is the clinically limiting factor. This investigation has suggested that a newly developed histogram technique that uses internal reference tissues close to the spine may have greater precision than the conventional techniques that depend on a peripherally located external phantom for calibration. The histogram technique combines computer power with physics to eliminate reproducibility errors introduced by use of an external reference phantom and to minimize ROI repositioning error. Further comparative and long-term studies are necessary to confirm these preliminary results. However, this technique appears to have promise for increasing the precision of QCT bone measurements toward clinically applicable levels.

\section{REFERENCES}

1. Reinbold WD, Genant HK, Reiser UJ, et al: Bone mineral content in early-postmenopausal and postmenopausal osteoporotic women: Comparison of measurement methods. Radiology 160:469-478, 1988

2. Genant HK, Steiger P, Block JE, et al: Quantitative computed tomography: Update 1987. Calcif Tissue Int 41:179-186, 1987

3. Health and Public Policy Committee, American College of Physicians: Bone mineral densitometry. Ann Intern Med 107:932-936, 1987

4. Mazess RB: Bone density in diagnosis of osteoporosis: Thresholds and breakpoints. Calcif Tissue Int 41:117-118, 1987

5. Cann CE, Genant HK, Kolb FO, et al: Quantitative computed tomography for prediction of vertebral fracture risk. Bone 6:1-7, 1985

6. Cann CE, Genant HK: Precise measurement of vertebral mineral content using computed tomography. J Comput Assist Tomogr 4:493-500, 1980

7. Goodsitt MM, Rosenthal DI, Reinus WR, et al: Two postprocessing $\mathrm{CT}$ techniques for determining the composition of trabecular bone. Invest Radiol 22:209-215, 1987

8. Merritt RB, Chernery SG: Quantitative CT measurements: The effect of scatter acceptance and filter characteristics on the EMI 7070. Phys Med Biol 31:55-63, 1986

9. McCullough EC: Factors affecting the use of quantitative information from a CT scanner. Radiology 124:99-107, 1977 
10. Rutherford RA, Pullan BR, Isherwood I: Measurement of effective atomic number and electron density using the EMI scanner. Neuroradiology 11:15-21, 1976

11. Hall FM, Davis MA, Baran DJ: Bone mineral screening for osteoporosis. N Engl J Med 316:212-214, 1987

12. Kalender WA, Klotz E, Suess C: Vertebral bone mineral analysis: An integrated approach with CT. Radiology 164:419-423, 1987

13. Imamura K, Masamichi F: Empirical beam hardening correction in the measurement of vertebral bone mineral content by computed tomography. Radiology 138:223-226, 1981

14. Meagher JM, Mote CD, Skinner HB: CT image correction for beam hardening using simulated projection data. Transactions of the 34th Annual Meeting of the Orthopaedic Research Society, Atlanta, 1988

15. Hemmingsson A, Jung B, Ytterbergh C: Dual energy computed tomography: Simulated monoenergetic and material-selective imaging. J Comput Assist Tomogr 10:490-499, 1986

16. Cann CE: Quantitative CT applications: Comparison of current scanners. Radiology 162:257-261, 1987

17. Goodsitt MM, Rosenthal DI: Quantitative computed tomography scanning for measurement of bone and bone marrow fat content: A comparison of single- and dual-energy techniques using a solid synthetic phantom. Invest Radiol 22:799-810, 1987

18. White DR: Tissue substitutes in experimental radiation physics. Med Phys 5:467-479, 1978

19. Glover GH, Pelc NJ: Nonlinear partial volume artifacts in X-ray computed tomography. Med Phys 7:238-248, 1980

20. Breatnach E, Robinson PJ: Repositioning errors in measurement of vertebral attenuation values by computed tomography. Br J Radiol 56:299-305, 1983

21. Cann CE: Quantitative CT for determination of bone mineral density: A review. Radiology 166:509-522, 1988

22. Laval-Jeantet AM, Roger B, Bouysse S, et al: Influence of vertebral fat content on quantitative CT density. Radiology 159:463-466, 1986

23. Mazess RB: Errors in measuring trabecular bone by computed tomography due to marrow and bone composition. Calcif Tissue Int 35:148-152, 1983

24. Rosenthal DI, Ganott MA, Wyshak G, et al: Quantitative computed tomography for spinal density measurement: Factors affecting precision. Invest Radiol 20:306-310, 1985 